\title{
The fixation of chromosomal rearrangements in a subdivided population with local extinction and colonization
}

\author{
Russell Lande
}

Department of Biophysics and Theoretical Biology, The University of Chicago, Chicago, Illinois 60637.

\begin{abstract}
The fixation of major chromosomal rearrangements with a heterozygote disadvantage is modelled as a simple example of Wright's shifting balance process of evolution in a strongly subdivided population. Chromosomal mutations with an inferior heterozygote become fixed in a local population (or deme) by random genetic drift and spreas by migration and colonization. Wright postulated a process of selective diffusion in which the numbers of emigrants and colonizers dispersed from a deme increase with the mean fitness of individuals in it. The present models show that interdeme selection during the spread of a mutation depends more on the capacity of the mutant to invade and become established in other demes than on selective diffusion, unless there is rapid local extinction and colonization. The intensity of interdeme selection is reduced by random local extinction and colonization, and when these processes are rapid (with no selective diffusion) the expected fixation rate of spontaneous mutations with a heterozygote disadvantage approaches that in a single isolated deme. Local extinction and colonization, and selection on the homozygotes, accelerate the spread of chromosomal mutations which are destined to be fixed.
\end{abstract}

\section{INTRODUCTION}

The shifting balance theory of evolution developed by Wright (1931, 1932, 1940, 1949, 1970, 1977) concerns the origin and spread of novel adaptations in geographically subdivided populations. The interaction of many genetic and environmental factors producing an intermediate optimum phenotype for a set of characters of an organism implies a large number of adaptive gene combinations, because many different genotypes can produce nearly the same optimum phenotype, with only minor differences in phenotype due to pleiotropic effects of the genes. There may also be more than one optimum phenotype or ecological niche available to a population. In Wright's conception a population is represented as a point on a multidimensional surface with many peaks and valleys. The height of this adaptive landscape is the mean fitness of individuals in the population, and the other dimensions are gene frequencies. Selection in a large population always moves the population uphill on the adaptive landscape, increasing the mean fitness, provided that the selective forces on pairs of genetic factors are weaker than their recombination rates so that genes at different loci are approximately combined at random in the population (near linkage equilibrium). The mean fitness in a population actually tends to increase under more general conditions (Nagylaki, 1976 chapter 8; Ewens, 1977 chapter 6).

To allow continuing adaptation by the attainment of new and higher adaptive peaks, Wright (1931, 1932, 1940) postulated a trial and error process of random genetic drift in small local populations (colonies or demes) which exchange at most a few migrants per generation with other local populations. Mutation and migration between genetically different populations maintain genetic variability within demes, one of which may occasionally move by random genetic drift away from an old adaptive peak and come under the influence of a new and possibly higher adaptive peak. Once an improved adaptation has been achieved by a local population, through selective diffusion this deme may disperse more migrants and colonists than other demes, thus imparting a further advantage in the spread of the novel adaptation by interdeme selection (Wright, 1940; $1970 ; 1977$ ). 
Wright's shifting balance theory of evolution contains many complexities, the theoretical consequences of which have never been analyzed in detail. One of these is the problem of spreading a polygenic adaptation by the selective diffusion of rare migrants into existing colonies where, upon crossing, they will be broken up by recombination (Wright, 1949). This difficulty is partially circumvented by major chromosomal rearrangements, such as centric fusions which link previously unlinked genes, and especially by inversions which suppress recombination within and around the inverted region.

However, in most outcrossing species major chromosomal rearrangements such as large inversions, centric fusions and fissions, and reciprocal translocations usually suffer a substantial heterozygote disadvantage due to the production of aneuploid gametes. Consequently they are selected against when in the minority and favored when in the majority (Wright, 1940; 1941). Exceptions to this rule are small inversions or centric fusions between telocentric chromosomes of nearly equal length, and paracentric inversions in Diptera, which consistently produce normal gametes (White, 1973).

The spontaneous rates of occurrence of different types of chromosomal rearrangements are of similar magnitude in a variety of species, around $10^{-4}$ to $10^{-3}$ per gamete per generation, with reciprocal translocations tending to predominate over inversions when the number of chromosomes is large (Lande, 1979). But in species which are predominantly outcrossing the fixation rate of inversions and centric fusions and fissions is much higher than that of reciprocal translocations. This is largely because the heterozygote disadvantage of reciprocal translocations, around 50 per cent, is much greater than that for most inversions and fusions or fissions, around a few to several per cent (Sturtevant, 1938; Spieth and Heed, 1972). Closely related species often differ by large inversions and asymmetrical fusions which appear to have created an appreciable frequency of aneuploid gametes in heterozygotes (White, 1973; 1978). The average fixation rate of major chromosomal rearrangements is about $10^{-7}$ to $10^{-6}$ per generation in vertebrate lineages, and roughly an order of magnitude slower in invertebrates (Bush et al., 1977; Lande, 1979; Imai et al., 1983).

To account for the spread and fixation of major chromosomal rearrangements which produce a substantial proportion of aneuploid gametes when heterozygous, it is necessary to postulate either (1) a deterministic advantage sufficient to outweigh the heterozygote disadvantage, such as selection on position effects, meiotic drive, or linkage with a favorable combination of genes, or (2) stochastic processes such as local establishment at high frequency by random genetic drift and spread by migration and colonization.

Wright $(1940 ; 1941)$ emphasized that small effective sizes of local populations facilitate the fixation of major chromosomal rearrangements with a substantial heterozygote disadvantage. Random genetic drift provides a mechanism for the local fixation of rearrangements whch are deleterious when rare but advantageous when common. Wright (1940) noted that in geographical regions where local populations are subject to frequent extinction and recolonization by a few individuals from a neighboring colony, the effective size of local populations may be much smaller than their average actual size, and that after sufficient time all of the colonies in a wide area are likely to be descended from a single ancestral colony. Formalizing this model, Lande (1979) showed that in a strongly subdivided population with local extinction and recolonization from a neighboring colony the expected fixation rate of strictly underdominant mutations is determined by the effective size of local populations and is independent of the number of colonies. Lande also argued that for mutations with a substantial heterozygote disadvantage high rates of local extinction and recolonization accelerate the spread of a rearrangement in homozygous forms. Rapid local extinction and colonization has been observed in many species across all or part of their geographical range, often at rates in the range of $10^{-3}$ to $10^{-1}$ per generation (Simberloff, 1974; Schoener, 1983; Diamond, 1984). Even in species with large stable central populations, local extinction and colonization may often occur at the species borders with fluctuations in weather and the densities of interacting species, or at least once every $5 \times 10^{5}$ years during the Pleistocene glaciations.

Slatkin (1977) and Maruyama and Kimura (1980) modeled the maintenance of selectively neutral variation by mutation in a subdivided population with local extinction and colonization. Nagylaki (1980), Slatkin (1981) and Maruyama $(1974,1983)$ analyzed the spread of a selected mutation in a subdivided population. All of the previous models have neglected one or more of the potentially important mechanisms in Wright's shifting balance theory. In particular, there have been no stochastic models of the process of selective diffusion, in which the local populations with 
the highest mean fitness gain an advantage in interdeme selection by dispersing more emigrants and colonizers. The present paper outlines a simple model of Wright's shifting balance theory applied to the evolution of major chromosomal rearrangements.

\section{DETERMINISTIC MODELS}

In a large population distributed with a uniform density in space a rare mutant gene with an advantage both as a heterozygote and a homozygote will spread through the population in a deterministic wave of advance first described by Fisher (1937). In contrast, a mutation with a heterozygote disadvantage and a homozygote advantage cannot spread through a continuously distributed population unless it is initially established at a high frequency in a local region of sufficient size (Fife and McLeod, 1977; Aronson and Weinberger, 1978; Barton, 1979). Furthermore, the wave of advance of an advantageous underdominant mutation cannot pass through a major barrier to gene flow or a region of low population density (Bazykin, 1969; Barton, 1979). This occurs because when migration rates between two adjacent regions are low the mutation is initially rare when crossing the boundary and is kept rare by selection against heterozygotes. Thus, strongly subdivided population structures which favor the local establishment of underdominant mutations by random genetic drift are not conducive to their spread by deterministic processes.

The effect of a barrier to migration in preventing the spread of an underdominant gene can be illustrated most simply in the case of an advantageous mutant genotype invading an island on which an inferior homozygote is initially fixed. A proportion $m$ of the island population is replaced each generation by advantageous mutant homozygotes. Mating is assumed to be at random on the island, with overlapping generations. Ignoring departures from Hardy-Weinberg frequencies of the genotypes by assuming that selection and migration are weak, the rate of change of the frequency of the advantageous mutation on the island is approximately

$$
d p / d t=p(1-p)\left[-s(1-2 p)+s^{\prime} p\right]+m(1-p)
$$

where $s$ is the selective disadvantage of the heterozygote, $s^{\prime}$ is the selective advantage of the mutant homozygote, and time, $t$, is measured in generations. At equilibrium the frequency of the mutant on the island is either $p=1$, which is stable, or

$$
p=\left[1 \pm \sqrt{1-8(m / s)\left(1+s^{\prime} / 2 s\right)}\right] /\left[4\left(1+s^{\prime} / 2 s\right)\right] .
$$

The smaller of the last two solutions is a stable equilibrium which would be achieved starting from a low frequency of the mutant on the island, and the larger of the two solutions is an unstable equilibrium. For migration rates lower than the threshold value, $m<s / 8\left(1+s^{\prime} / 2 s\right)$, both of the equilibria in (lb) exist and the advantageous mutation cannot invade the island population.

A similar effect occurs in a linear array of colonies of equal size formed by contact between two chromosomal races. Each colony exchanges a proportion $m / 2$ of its population with both of the adjacent colonies. Assuming the contact zone between the races is between colonies 0 and 1 , the initial condition is $p_{i}=1$ for $i \leqq 0$ and $p_{i}=0$ for $i \geqq 1$. The rate of change in frequency of the advantageous gene arrangement in the $i$ th colony is approximately

$$
\begin{aligned}
d p_{i} / d t= & p_{i}\left(1-p_{i}\right)\left[-s\left(1-2 p_{i}\right)+s^{\prime} p_{i}\right] \\
& +\frac{1}{2} m\left(p_{i-1}+p_{i+1}\right)-m p_{i} .
\end{aligned}
$$

Provided that the migration rate is much less than the heterozygote disadvantage ( $m \ll$ $\left.2 \min \left[s, s+s^{\prime}\right]\right)$ the equilibrium frequency of the mutant gene arrangement in any given colony is significantly influenced by only one of its nearest neighbors. Starting at the boundary between the races and sequentially solving for the chromosome frequency in each colony gives the equilibrium geographical pattern as approximately

$$
p_{i}= \begin{cases}1-\left[m / 2\left(s+s^{\prime}\right)\right]^{1-i} & \text { for } i \leqq 0 \\ {[m / 2 s]^{i}} & \text { for } i \geqq 1 .\end{cases}
$$

There is a large discontinuity in the frequency of the underdominant mutation at the boundary between the races, with a geometric decrease in the frequency of the rare chromosomal arrangement on each side of the boundary. The stability of this equilibrium to small perturbations is guaranteed by Karlin and McGregor's (1972) theory of small parameters, since the initial condition is a stable equilibrium in the absence of migration.

From these simple models it appears that temporal changes in population structure would favor the initial establishment of underdominant mutations by random genetic drift in a local population and their subsequent rapid spread to other regions. 
These could consist either of large and possibly synchronous fluctuations in population density over a wide area so that periods of population subdivision alternate with periods of continuous distribution, or permanent subdivision with rapid local extinction and colonization (Wright, 1931; $1940 ; 1970)$. The latter type of population structure is analyzed here to assess the potential for evolution of adaptive underdominant mutations in a strongly subdivided population.

\section{STOCHASTIC MODELS}

\section{(i) Assumptions}

In a subdivided population composed of colonies or demes of effective size $N_{e}$ and average actual size $N$, mating is assumed to be random within each colony, except that in each generation a small proportion $m$ of the individuals in each colony are replaced by migrants. For major chromosomal rearrangements with an inferior heterozygote, which are deleterious when rare, each colony will tend to maintain one karyotype in high frequency with rare variants introduced by mutation and immigration. Assuming that every spontaneous rearrangement is one not currently existing in the population, the fixation of a particular rearrangement in a strongly subdivided population can be separated into two events: (1) spontaneous occurrence and fixation or establishment at a stable high frequency in a single colony by random genetic drift and natural selection, and (2) spread to other areas by invasion of existing colonies and founding of new colonies. Following a local extinction, recolonization is accomplished by individuals from a single neighboring colony, which allows underdominant mutations to spread in homozygous form instead of by immigration and random genetic drift against the force of selection in every colony.

A description of this type requires the condition that the expected time a mutation segregates on the way to fixation (or establishment) within a colony in much less than the expected time between the arrival of immigrant genotypes destined to be established. The great majority of mutations with an appreciable heterozygote disadvantage are expected to be lost within a few or several generations, whereas the expected number of generations during which such mutations segregate on the way to fixation is less than that for a selectively neutral mutation, $4 N_{e}$ (Kimura and Ohta, 1969; Lande, 1979). Letting $U$ represent the probability of local fixation or establishment of a new mutation in a single colony, the expected time between the arrival of immigrant mutations destined to be established can be as great as $1 /(2 \mathrm{NmU})$ only if all immigrant gene arrangements are not of the prevailing type in the colony. Since the probability of local fixation or establishment of an underdominant mutant is less than that for a neutral mutation, $U<1 / 2 N$, the foregoing condition will be satisfied if the effective number of immigrant individuals entering a colony is much less than one per four generations on average, $N_{e} m \ll{ }_{4}^{1}$. This degree of genetic isolation between colonies is sufficient for immigration to be negligible in calculating the fixation or establishment probability of a single mutation introduced into a colony (Lande, 1979). Similarly we suppose that the spontaneous rearrangement rate for any region of the genome is low, $N_{e} \mu \ll \frac{1}{4}$, so that different rearrangements of the same chromosome segregating within a colony do not interfere with each other (Guess and Ewens, 1972).

The effective size of a local population, $N_{e}$, may be considerably smaller than the average actual size, $N$, especially when there are marked fluctuations in numbers with time (Wright, 1931; 1969 chapter 8). Reduction of population size through recolonization by a few individuals from a neighboring colony would also contribute to reducing $N_{e}$ so that frequent local extinction and colonization may be associated with small effective sizes of local populations (Wright, 1940).

Two models for the spatial configuration of the population will be analyzed. These are the circular stepping stone model, in which the colonies are arranged in a circle and migrants are exchanged only between adjacent pairs of colonies, and the island model in which migrants disperse randomly to all colonies. At first it is assumed that regardless of genotypic composition, each colony sends out the same number of emigrants as any other colony; later this assumption is relaxed when the number of emigrants from a colony is taken to be proportional to the mean fitness of individuals in it. These alternative forms of dispersal correspond to models of "soft" and "hard" selection (Christiansen, 1975), which respectively describe local versus global resource limitation of population density.

\section{(ii) Probabilities and rates of fixation}

In both the circular stepping stone model and the island model colonies are interchangeable, in that the probability of fixation of a new mutant does not depend on the colony in which it arises. The expected fixation rate per generation of a class of 
chromosomal rearrangements with the same selection coefficients can be written for both models as

$$
r=R n P .
$$

In this formula $n$ is the number of colonies and $R$ is the rate of local fixation or establishment by spontaneous rearrangements in a single colony. $P$ is the probability that a given rearrangement which is initially fixed or established in a single colony will spread and become completely fixed in the entire population. Because the colonies are interchangeable in the sense described above, it is evident that when there is no selection for or against rearrangement homozygotes each colony has an equal chance of spreading its gene arrangement throughout the entire population, hence $P=$ $1 / n$ and the expected fixation rate in the entire population is equal to that in a single isolated colony, $r=R$ (Lande, 1979; Slatkin, 1981). In general $n P$ is the probability of complete fixation of a mutation which is initially fixed or established at high frequency in a single colony, relative to that of a strictly underdominant (or neutral) mutation for which the two homozygotes are equally fit.

The rate of local fixation or establishment of new rearrangements within a colony is equal to the number of spontaneous rearrangements, $2 N \mu$, times the probability of local fixation or establishment starting from a single mutant, $U$,

$$
R=2 N \mu U
$$

in which $\mu$ is the spontaneous rate for a class of rearrangements with the same selection coefficients. A formula for the fixation probability of a new mutation in a single population was given by Kimura $(1962,1964)$,

$$
U=\int_{0}^{1 / 2 N} G(x) d x / \int_{0}^{1} G(x) d x
$$

where

$$
G(x)=\exp \left\{4 N_{e}\left[s x(1-x)-s^{\prime} x^{2} / 2\right]\right\} .
$$

When selection on underdominant mutations is not very strong the numerator in $(3 \mathrm{c})$ can be approximated as $1 / 2 N$ and the denominator can be evaluated to yield

$$
U=\frac{(2 / N) \exp \left\{-N_{e} s^{2} / b\right\} \sqrt{N_{e} b / \pi}}{\operatorname{erf}\left\{\left(s+s^{\prime}\right) \sqrt{N_{e} / b}\right\}+\operatorname{erf}\left\{s \sqrt{N_{e} / b}\right\}}
$$

in which $b=s+s^{\prime} / 2$ and $\operatorname{erf}\{k\}=$ $(2 / \sqrt{\pi}) \int_{0}^{k} \exp \left\{-y^{2}\right\} d y$ is tabulated in Abramowitz and Stegun (1972). It now remains to calculate the probability, $P$, that a mutation will spread and reach complete fixation, given that it is initially fixed or established in a single colony.

With low migration rates $\left(N_{e} m \ll \frac{1}{4}\right)$ the probability that the genotype of an immigrant homozygous for a mutant chromosomal arrangement will become fixed or established at a high stable frequency in a colony where the standard arrangement prevails is from diffusion theory (Kimura, $1962 ; 1964)$

$$
\int_{0}^{1 / N} G(x) d x / \int_{0}^{1} G(x) d x .
$$

When individual selection on the mutant is not very strong this is approximately $2 U$, which is twice the probability of local fixation starting from one copy of the mutation. The chance of the reverse transition, that the genotype of an immigrant homozygous for the standard gene arrangement will become fixed or established in a colony where a mutant gene arrangement is established is

$$
\int_{1-1 / N}^{1} G(x) d x / \int_{0}^{1} G(x) d x .
$$

Under the assumption of weak individual selection this is approximately $2 U \exp \left\{-2 N_{e} s^{\prime}\right\}$ (Slatkin, 1981). The probability of successful invasion and fixation or establishment of a migrant genotype in a colony of a different genotype will be referred to as the invasiveness of the migrant genotype.

With migration and/or local extinction and recolonization in a strongly subdivided population $\left(N_{e} m \ll \frac{1}{4}\right)$, the spread of a mutation which has become fixed or established in a single colony is nearly equivalent to a random walk on the number of colonies in which the mutant is fixed or established (Lande, 1979; Slatkin, 1981). The relative rates of increasing and decreasing the number of colonies in which a mutation is fixed or established, denoted respectively as $u$ and $v$, determine the probability of fixation in the entire population starting from fixation or establishment in a single deme: $P=1 / n$ if $u=v$ as stated above, and from Slatkin (1981)

$$
P=[1-v / u] /\left[1-(v / u)^{n}\right] \quad \text { if } u \neq v .
$$

The forward and reverse transition rates $u$ and $v$ will now be specified for different patterns of selection.

Soft selection. First consider a circular array of $n$ colonies, with migration between them at a rate of $m$ per individual per generation so that $\mathrm{Nm} / 2$ individuals are exchanged between every adjacent pair of colonies each generation. In this model of "soft" selection all colonies are assumed to be 
equivalent in terms of the number of emigrants and colonizers dispersed per generation. Each colony also becomes extinct with probability $\lambda$ per generation, and there is an equal chance of being recolonized by individuals from either of the two adjacent colonies. A chromosomal mutation which is initially established at high frequency in a single colony can spread in two directions in the circular array of colonies. Assuming that the rates of successful invasion and establishment by migrants, $2 N m U$ and $2 N m U \exp \left\{-2 N_{e} s^{\prime}\right\}$, and the rate of local extinction and recolonization, $\lambda$, are each much smaller than unity the forward and reverse transition rates for the number of demes in which a particular mutant predominates are approximately

$$
\begin{aligned}
& u=2 N m U+\lambda \\
& v=2 N m U \exp \left\{-2 N_{e} s^{\prime}\right\}+\lambda .
\end{aligned}
$$

When extinction and colonization are slow compared to spread of genotypes by invasion of existing demes, the ratio $v / u$ approaches $\exp \left\{-2 N_{e} s^{\prime}\right\}$ as found by Slatkin (1981). When random extinction and recolonization is relatively rapid the ratio $v / u$ approaches unity, $P$ approaches $1 / n$, and from (3a) the fixation rate in the entire population approaches that in a single deme. A large number of demes promotes apparent interdeme selection in favor of the most advantageous homozygote, but a high rate of random local extinction and recolonization decreases the intensity of interdeme selection. These features of the model are shown numerically in table 1 .

In the island model with "soft" selection, migration occurs at random among every pair of colonies. Each colony becomes extinct with probability $\lambda$ per generation and is recolonized at random from a pool of colonizing propagules to which each colony in the population contributes equally. The rates of increase and decrease in the number of colonies in which a mutant is fixed or established at high frequency are $n X(1-X)$ times those in the circular stepping stone model, where $X$ is the proportion of colonies in which a particular mutant is fixed or established. The ratio $v / u$ and the probability $P$ are thus the same in both models of population structure. Slatkin (1981) previously showed that the fixation probability of a new mutant in a strongly subdivided population is independent of its geographical structure, provided that the matrix of migration rates among the colonies is symmetric.

Hard selection. Wright's shifting balance theory of evolution postulates a process of "selective diffusion" in which demes containing individuals
Table 1 The probability of complete fixation of a mutation which is initially fixed in one colony, relative to that of a strictly underdominant (or neutral) mutation, $n P$. Selection is "soft" so that the number of emigrants and colonizing propagules dispersed from a colony is independent of its genotypic composition. The number of colonies is $n$ and the rate of local extinction and recolonization is $\lambda$ per colony per generation. Numerical values were computed from approximate formulae in the text with $N_{c}=50$ individuals per colony, $N m=0.02$ immigrants per colony

\begin{tabular}{|c|c|c|c|c|c|}
\hline \multicolumn{6}{|c|}{ Mutant homozygote disadvantage $\left(s^{\prime}=-0.01\right)$} \\
\hline$\lambda$ & $n=$ & 10 & $10^{2}$ & $10^{3}$ & $10^{4}$ \\
\hline $10^{-4}$ & & $7.9 \times 10^{2}$ & $3 \times 10^{17}$ & $1 \times 10^{-170}$ & $4 \times 10^{-1723}$ \\
\hline $10^{3}$ & & 0.72 & $6.8 \times 10^{-3}$ & $4 \times 10^{29}$ & $3 \times 10^{-289}$ \\
\hline $10^{2}$ & & 0.97 & 0.68 & $4.3 \times 10^{-3}$ & $3 \times 10^{31}$ \\
\hline $10^{1}$ & & 0.997 & 0.96 & 0.67 & $4.1 \times 10^{-3}$ \\
\hline
\end{tabular}
per generation and a heterozygote disadvantage of $s=0.05$

\begin{tabular}{|c|c|c|c|c|c|}
\hline$\lambda$ & $n=$ & 10 & $10^{2}$ & $10^{3}$ & $10^{4}$ \\
\hline $10^{-4}$ & & $2 \cdot 90$ & $27 \cdot 9$ & 279 & 2786 \\
\hline $10^{-3}$ & & $1 \cdot 23$ & $4 \cdot 66$ & $46 \cdot 2$ & 462 \\
\hline $10^{-2}$ & & 1.02 & 1.27 & 4.98 & $49 \cdot 4$ \\
\hline $10^{-1}$ & & 1.002 & 1.02 & $1 \cdot 27$ & 5.01 \\
\hline
\end{tabular}

Mutant homozygote advantage $\left(s^{\prime}=+0.01\right)$

with a relatively high mean fitness disperse more migrants, and more colonizing propagules, than the average deme. In models of "hard" selection the number of emigrants from a local population is assumed to be simply proportional to the mean fitness of individuals in it. For the circular stepping stone model of population structure the rates of increase and decrease in the number of demes in which a mutation is fixed or established are then approximately

$$
\begin{aligned}
& u=\left(1+s^{\prime}\right)\left[2 N m U+\lambda /\left(1+s^{\prime} / 2\right)\right] \\
& v=2 N m U \exp \left\{-2 N_{e} s^{\prime}\right\}+\lambda /\left(1+s^{\prime} / 2\right) .
\end{aligned}
$$

The quantity dividing $\lambda$ in these expressions is a normalization factor that guarantees the rate of recolonization is equal to the rate of local extinction, which is assumed to be independent of the genotypic composition of a deme. However, in the present model of hard selection the average migration rate between colonies is allowed to change as a mutation spreads through the population. The detailed mechanism by which the numbers of migrants and colonizers are regulated under hard selection makes little difference in the probability of complete fixation, provided that selection on the mutant homozygote is weak, $\left|s^{\prime}\right| \ll 1$, in which case the ratio $v / u$ is approximately $1 /\left(1+s^{\prime}\right)$ times as large as under soft selection. In the island model of population structure the transition rates for the number of demes occupied by a mutation are 
$n X(1-X)$ times as large as in the foregoing equations.

In both the circular stepping stone and island models with selective diffusion, high rates of local extinction and colonization produce a ratio $v / u$ approaching $1 /\left(1+s^{\prime}\right)$ instead of unity as under soft selection, because relative rates of recolonization differ in local populations where different genotypes prevail. This provides a further advantage in interdeme selection to demes in which an individually advantageous mutation is fixed or established at high frequency. Numerical examples of fixation probabilities of a mutation initially fixed or established in a single deme and which can spread by selective diffusion are given in table 2 .

Table 2 The probability of complete fixation of a mutation which is initially fixed in one colony, relative to that of a strictly underdominant (or neutral) mutation, $n P$. There is selective diffusion ("hard" selection) such that the number of emigrants and colonizing propagules dispersed from a colony is proportional to the mean fitness of individuals in it. Other parameters are the same as in table 1

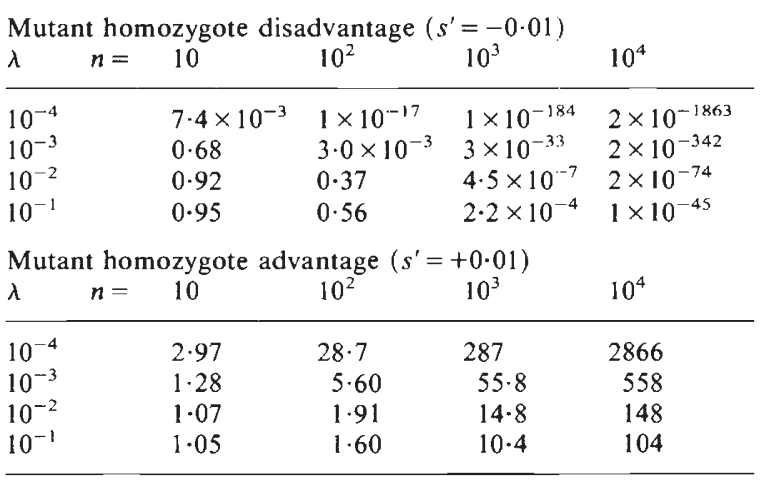

Comparison of tables 1 and 2 for soft and hard selection demonstrates that selective diffusion increases the apparent intensity of interdeme selection. However, even with selective diffusion ("hard" selection) the apparent interdeme selection is attributable mainly to differences in the invasiveness of the mutant and standard homozygotes, rather than differential productivity of the demes, unless the rate of local extinction and colonization is much higher than the rate of spread by migration into existing demes.

\section{(iii) Expected time to fixation}

Strict underdominance. When the mutant and wild type homozygotes are equally fit $\left(s^{\prime}=0\right)$, there is little distinction between "soft" and "hard" selection on underdominant mutations in a strongly subdivided population, since each of the colonies will be nearly homozygous almost all of the time. In this case there is equality of the rates of forward and reverse transitions in the number of demes where a particular mutation is fixed or established at high frequency. In the circular stepping stone model $u=v=2 \mathrm{Nm} U+\lambda$, and in the island model these rates are $n X(1-X)$ times as large, where $X$ is the proportion of demes in which a particular mutation is fixed or established. For both geographical structures $P=1 / n$ and the fixation rate of strictly underdominant mutations in the entire population is approximately the same as that in a single deme, $r=R$.

A formula from diffusion theory developed by Kimura and Ohta (1969) can be employed to calculate the expected number of generations until complete fixation of a mutant starting from local fixation or establishment at high frequency in a single deme (Slatkin, 1981), using the mean and variance of the change per generation in $X$, the proportion of demes in which a particular mutant is fixed or established at high frequency, as 0 and $8 \mathrm{NmU} / n^{2}$ respectively in the circular stepping stone model, and $n X(1-X)$ times as large in the island model. For the circular stepping stone model the expected time until fixation is approximately

$$
\bar{T}_{1}=\left(n^{2}-1\right) / 6[2 N m U+\lambda]
$$

and for the island model

$$
\bar{T}_{1}=2(n-1) /[2 N m U+\lambda] .
$$

The expected time to complete fixation of a strictly underdominant mutation is faster in the island model than in the circular stepping stone model when $n \geqq 12$. In both models of the geographical structure of the population, increasing the rate of local extinction and colonization can markedly accelerate the spread of a strictly underdominant mutation which is destined to be completely fixed.

For example consider a strictly underdominant mutation $\left(s^{\prime}=0\right)$ with a heterozygote disadvantage of $s=0.05$ in a population with $n=1000$ demes and an effective number of $N_{e}=50$ individuals per deme with an average immigration rate of $\mathrm{Nm}=$ 0.02 individuals per deme per generation. In the absence of local extinction and colonization $(\lambda=$ $0)$ the expected time until complete fixation, starting from fixation or establishment in a single deme is $2.77 \times 10^{9}$ generations in the circular stepping stone model and $3.32 \times 10^{7}$ generations in the island model. With local extinction and recolonization at a rate of $\lambda=0.01$ per colony per generation 
the expected number of generations until complete fixation is reduced to $1.66 \times 10^{7}$ in the circular stepping stone model and $1.98 \times 10^{5}$ in the island model.

Selection on homozygotes. When there is appreciable selection for or against the mutant homozygote and the number of demes is not very small ( $n \geqq 10$ and $N_{e}\left|s^{\prime}\right|>1$ ), with no extinction and colonization $(\lambda=0)$, the expected number of generations until complete fixation of a mutant which is initially fixed in a single deme can be obtained from Kimura and Ohta's (1969) formula, with the mean and variance of the change per generation in $X$ approximated as $4 N m U(1-$ $\left.\exp \left\{-2 N_{e} s^{\prime}\right\}\right) / n$ and $4 N m U\left(1+\exp \left\{-2 N_{e} s^{\prime}\right\}\right) / n^{2}$ respectively in the circular stepping stone model, and both the mean and variance are $n X(1-X)$ times as large in the island model. Under soft selection with $\lambda=0$ in the circular stepping stone model the expected time to complete fixation is about

$$
\bar{T}_{1}=n / 4 N m U\left|1-\exp \left\{-2 N_{i} s^{\prime}\right\}\right|
$$

and in the island model the expected time to complete fixation is smaller by a factor of roughly $n^{-1} \int_{1 / n}^{1-1 / n} y^{1}(1-y)^{-1} d y=2 n^{-1} \ln (n)$ for $n \geqq 10$, hence

$$
\bar{T}_{1}=\ln (n) / 2 N m U\left|1-\exp \left\{-2 N_{e} s^{\prime}\right\}\right|
$$

in which $\ln (n)$ is the natural logarithm (base $e$ ) of $n$. Selective diffusion (hard selection) decreases the expected fixation times only slightly from those in equations (8), adding $s^{\prime}$ to the factor inside the absolute values. Comparison of equations (7) and (8) reveals that the spread of an underdominant mutation destined to be completely fixed is accelerated by selection for or against the mutant homozygote.

For a numerical example, consider a mutation with a homozygote advantage of $s^{\prime}=+0.01$ and a heterozygote disadvantage of $s=0.05$ in a population with $n=1000$ demes, $N_{c}=50$ individuals per deme and $N m=0.02$ immigrants per deme per generation on average, and no local extinction and colonization, $\lambda=0$. Under soft selection the expected timc to completc fixation with initial fixation or establishment in one deme is about $1.03 \times 10^{7}$ generations in the circular stepping stone model and $1.42 \times 10^{5}$ generations in the island model. With selective diffusion (hard selection) the corresponding fixation times are only slightly smaller, $9.88 \times 10^{6}$ and $1.36 \times 10^{5}$ generations respectively.

\section{DISCUSSION}

Local fixation or establishment of an underdominant mutation (with a substantial heterozygote disadvantage), such as a major chromosomal rearrangement, has an appreciable chance of occurring by random genetic drift only in a strongly subdivided population with small, nearly isolated demes (Wright, 1941; Lande, 1979). However, an underdominant mutation with a homozygote advantage cannot spread by a deterministic wave of advance through a strongly subdivided population once it is locally established at high frequency (Bazykin, 1969; Barton, 1979; and see equations 1 and 2 above). Wright (1931, 1940, 1970) discussed the importance of a fluctuating population structure, including alternating low and high density (spatial subdivision versus continuity) and local extinction and recolonization, to facilitate the shift of a widespread population from one adaptive peak to another possibly higher one.

The evolution of major chromosomal rearrangements in a strongly subdivided population with local extinction and colonization provides a simple example of Wright's shifting balance process, since every spontaneous rearrangement with a heterozygote disadvantage creates a new adaptive peak for the population which can be attained by: (1) random genetic drift across an adaptive valley, (2) selection up the new peak, and (3) spread by migration and colonization. The third phase of Wright's shifting balance process involves interdeme selection by selective diffusion in which demes containing individuals with relatively high mean fitness disperse more emigrants and colonizers than other demes.

An assessment of the shifting balance theory of evolution has been difficult because of the lack of stochastic models of interdeme selection involving selective diffusion. The present models incorporate random local extinction, and recolonization by individuals from a single neighboring deme as described by Wright (1940, 1941). This conforms to the "propagule pool" model of recolonization, which is more conducive to interdeme selection than the "migrant pool" model where colonizing groups are formed from a random sample of the entire population (Wade, 1978).

Under "soft" selection each deme is assumed to disperse the same numbers of emigrants and colonizers, and any apparent interdeme selection (manifest as differential survival or proliferation of genotypes from different demes) is a direct outcome of individual selection and the stochastic processes of random genetic drift and random local 
extinction and recolonization. Equations (4) and (5) and the numerical examples in table 1 show that with soft selection the fixation probability of an advantageous underdominant mutation which is initially fixed in a single deme can greatly exceed that of a strictly underdominant (or neutral) mutant, especially when there are many demes in the population. Thus in a large, strongly subdivided population apparently intense interdeme selection can occur even in the absence of selective diffusion, due to the different invasiveness of the genotypes, i.e., variation in the ability of genotypes to invade and become fixed or established at high frequency in demes of another genotype. This mechanism of interdeme selection (the third phase in the shifting balance process), has been briefly referred to by Wright (1970, p. 20), who states that "selective peaks ... of significance in phase 3 are those characterized by rapid population growth and high dispersive and invasive capacities".

With selective diffusion or "hard" selection the numbers of emigrants and colonizing propagules dispersed from a deme are assumed to be proportional to the mean fitness of individuals in the deme. In this case an advantageous underdominant mutation which is initially fixed in a single deme has a greater chance of spreading throughout a strongly subdivided population than a strictly underdominant (or neutral) mutant, not only because of the greater invasiveness of the advantageous mutant homozygote, but also because of the dispersal of more emigrants and a higher rate of recolonizing neighboring demes which become extinct. Comparison of formulae (6) and (7) and tables 1 and 2 reveals however that the extra advantage in interdeme selection produced by selective diffusion is not very great unless there are many demes with frequent local extinction and recolonization. Therefore in many situations the spread of an underdominant mutation with a homozygote advantage may be attributable more to its high invasive capacity than to selective diffusion.

In both soft and hard selection regimes the fixation probability of a mutation in a strongly subdivided population is independent of the geographical structure of the population, as shown by Slatkin (1981) for populations under soft selection with a symmetric migration matrix and no extinction or colonization. High rates of random local extinction and recolonization dramatically decrease the apparent intensity of interdeme selection, whether selection is soft or hard. With soft selection and rapid local extinction and recolonization the expected fixation rate of spontaneous mutations in the entire population approaches that in a single isolated deme, as implied by Wright (1940) and argued by Lande (1979). Under hard selection, with selective diffusion influencing the recolonization process, rapid local extinction and colonization decrease the intensity of interdeme selection, but the fixation rate of underdominant mutations in the entire population does not approach that in a single deme. Nevertheless, even with selective diffusion the effective size of a strongly subdivided population with respect to the fixation of major chromosomal rearrangements may be orders of magnitude smaller than the average actual size.

Following the initial fixation or establishment in one deme, the further spread of a strictly underdominant mutation destined to be fixed in the entire population is greatly accelerated by rapid local extinction and colonization, which allows underdominant mutations to spread in homozygous form instead of by migration and random genetic drift against the force of natural selection when rare in each deme (equations 7). Natural selection for or against the mutant homozygote also markedly accelerates the spread of an underdominant mutation which is destined to be completely fixed (equations 8).

Acknowledgments I thank N. Barton, M. Slatkin, and B. Walsh for comments on the manuscript and T. Nagylaki, M. Wade, D. Walton and especially Sewall Wright for helpful discussions. This work was supported by Contract No. DE-AC0281 ER60014 with the U.S. Department of Energy.

\section{REFERENCES}

ABRAMOWITZ, M. AND STEGUN, I. A. (EDS.). 1972. Handbook of Mathematical Functions. Dover, New York.

ARONSON, D. G. AND WEINBERGER, H. F. 1978. Multidimensional nonlinear diffusion arising in population genetics. Adv. in Math., 30, 33-76.

BARTON, N. H. 1979. The dynamics of hybrid zones. Heredity, $43,341-359$.

BAZYKIN, A. D. 1969. Hypothetical mechanism of speciation. Evolution, 23, 685-687.

BUSH, G. I., CASE, S. M., WILSON, A. C. AND PATTON, J. L. 1977. Rapid speciation and chromosomal evolution in mammals. Proc. Natl. Acad. Sci. U.S.A., 74, 3942-3946.

CHRISTIANSEN, F. B. 1975. Hard and soft selection in a subdivided population. Amer. Natur., 109, 11-16.

DIAMOND, J. M. 1984. "Normal" extinctions of isolated populations. (in press) In Nitecki, M. (ed.) Extinctions, Univ. of Chicago Press, Chicago.

EWENS, W. J. 1977. Mathematical Population Genetics. SpringerVerlag, New York.

FIFE, P. (. AND MCI.fOOI), J. B. 1977. The approach of solutions of nonlinear diffusion equations to travelling front solutions. Arch. Rational Mechanics and Analysis, 65, 335-361. 
FISHER, R. A. 1937. The wave of advance of advantageous genes. Ann. Eugenics, 7, 355-369.

(iUf.SS, H. A. ANI) EWENS, W. J. 1972. Theoretical and simulation results relating to the neutral theory. Theor. Pop. Biol, 3, 434-447.

IMAI, H. T., MARUYAMA, T. AND CROZII:R, R. H. 1983. Rates of mammalian karyotype evolution by the karyograph method. Amer. Natur., 121, 447-488.

KARLIN, S. AND MCGRE(iOR, J. 1972. Application of method of small parameters to multi-niche population genetic models. Theor. Pop. Biol., 3, 186-209.

KIMURA, M. 1962. On the probability of fixation of a mutant gene in a population. Genetics, 47, 713-719.

KIMURA, M. 1964. Diffusion models in population genetics. $J$. Applied Probability, 1, 177-232.

KIMURA, M. ANDOHTA, T. 1969. The average number of generations until fixation of a mutant gene in a finite population. Genetics, 61, 763-771.

LANIDE, R. 1979. Effective deme sizes during long-term evolution estimated from rates of chromosomal rearrangement. Evolution, 33, 234-251.

MARUYAMA, T. 1974. A simple proof that certain quantities are independent of the geographical structure of population. Theor. Pop. Biol., 5, 148-154.

MARUYAMA, F. 1983. Stochastic theory of population genetics. Bull. Math. Biol., 45, 521-554.

MARUYAMA, T. AND KIMURA, M. 1980. Genetic variability and effective population size when local extinction and recolonization are frequent. Proc. Nall. Acad. Sci. U.S.A., $77,6710-6714$.

NAGYLAK1, T. 1976. Selection in One-and Two-locus Systems. Springer-Verlag, New York.

NAGiYLAKI, T. 1980. The strong-migration limit in geographically structured populations. J. Math. Biol., 9, 101-114.

SCHOENER, I. W. 1983. Rate of species turnover decreases from lower to higher organisms: a review of the data. Oikos, 41, 372-377.
SLATKIN, M. 1977. Gene flow and genetic drift in a species subject to frequent local extinctions. Theor. Pop. Biol., 12, $253-262$

SLATKIN, M. 1981. Fixation probabilities and fixation times in a subdivided population. Evolution, 35, 447-488.

SIMBERLOFF, 1). S. 1974. Equilibrium theory of island biogeography. Ann. Rev. Ecol. Syst., 5, 161-182.

SPIETH, H. T. AND HEED, W. B. 1972. Experimental systematics and ecology of Drosophila. Ann. Rev. Ecol. Syst., 3, 269-288.

STURTEVANT, A. H. 1938. Essays on evolution. III. On the origin of interspecific sterility. Quart. Rev. Biol., 13, 333335 .

WADE, M. J. 1978. A critical review of the models of group selection. Quart. Rev. Biol., 53,101-114.

WHITE, M. J. D. 1973. Animal Cytology and Evolution. Wm. Clowes and Sons, London.

WhiTe, M. J. D. 1978. Modes of Speciation. Freeman, San Francisco.

WRIGHT, S, 1931. Evolution in Mendelian populations. Genetics, 16, 97-159.

WRIGHT, S. 1932. The roles of mutation, inbreeding, crossbreeding and selection in evolution. Proc. Sixth International Congress of Genetics, 1, 356-366.

WRIGHT, S. 1940. Breeding structure of populations in relation to speciation. Amer. Natur., 74, 232-248.

WRIGHT, S. 1941. On the probability of fixation of a reciprocal translocation. Amer. Natur., 75, 513-522.

WRIGiHT, S. 1949. Population structure in evolution. Proc. Amer. Philos. Soc., 93, 471-478.

WRIGHT, S. 1969. Evolution and the Genetics of Populations, Vol. 2. The Theory of Gene Frequencies. Univ. of Chicago Press, Chicago.

WRIGHT, s. 1970. Random drift and the shifting balance theory of evolution. In Kojima, K. (ed.), Mathematical Topics in Population Genetics. Springer-Verlag, Berlin, pp. 1-31.

WRIGHT, S. 1977. Evolution and the Genetics of Populations, Vol. 3. Experimental Results and Evolutionary Deductions. Univ. of Chicago Press, Chicago. 